\title{
Heat fluctuations and fluctuation theorems in the case of multiple reservoirs
}

\author{
Hans C. Fogedby* \\ Department of Physics and Astronomy, \\ University of Aarhus, Ny Munkegade \\ 8000 Aarhus C, Denmark \\ and \\ Niels Bohr Institute, Blegdamsvej 17 \\ 2100 Copenhagen Ø, Denmark \\ Alberto Imparatd \\ Department of Physics and Astronomy \\ University of Aarhus, Ny Munkegade \\ 8000 Aarhus C, Denmark
}

\begin{abstract}
We consider heat fluctuations and fluctuation theorems for systems driven by multiple reservoirs. We establish a fundamental symmetry obeyed by the joint probability distribution for the heat transfers and system coordinates. The symmetry leads to a generalisation of the asymptotic fluctuation theorem for large deviations at large times. As a result the presence of multiple reservoirs influence the tails in the heat distribution. The symmetry, moreover, allows for a simple derivation of a recent exact fluctuation theorem valid at all times. Including a time dependent work protocol we also present a derivation of the integral fluctuation theorem.
\end{abstract}

PACS numbers: 05.40.-a,05.70.Ln

*Electronic address: fogedby@phys.au.dk

${ }^{\dagger}$ Electronic address: imparato@phys.au.dk 


\section{INTRODUCTION}

There is a current interest in the thermodynamics and statistical mechanics of fluctuating systems in contact with heat reservoirs and driven by external forces. The current focus stems from the recent possibility of direct manipulation of nano systems and biomolecules. These techniques permit direct experimental access to the probability distribution functions for the work or for the heat exchanged with the environment [1 13]. These methods have also yielded access to the experimental verification of the recent fluctuation theorems which relate the probability of observing entropy-generated trajectories with that of observing entropy-consuming trajectories [6, 14 32].

In the present paper we address the issue of heat fluctuations and fluctuation theorems in the case of a system coupled to multiple reservoirs. The case of a system driven by two heat reservoirs, both in the case of one degree of freedom and in the case of many degrees of freedom, has been discussed extensively, see e.g. [33, 34], whereas the case of multiple reservoirs seems to have received less attention. In the case where a system is coupled to many reservoirs the heat flows exhibit a more complicated pattern which will influence the heat distribution.

We characterise the heat transfers to the system by the vector quantity $\mathbf{Q}$ with components $\left\{Q_{n}\right\}$, referring to the n-th heat reservoir maintained at temperature $T_{n}=1 / \beta_{n}$; we set Boltzmann's constant $k_{\mathrm{B}}=1$. We, moreover, assume that the reservoirs are independent. Noting that the transfer of heat also influences the internal state of the system characterised by the coordinate $x$, the central quantity is the joint distribution $P(\mathbf{Q}, x t)$. For simplicity we consider a single degree of freedom $x$; the generalisation to many degrees of freedom is straightforward and discussed at the end of the paper.

Assuming that the system is initially in the state $x_{0}$ at time $t_{0}$ and that the sampled heat vanishes at $t_{0}$, the joint distribution $P\left(\mathbf{Q}, x t, x_{0} t_{0}\right)$ describes the transition of the system from the initial state $x_{0}$ at time $t_{0}$ to the final state $x$ at time $t$ in contact with multiple heat reservoirs transferring the heat $\mathbf{Q}$ to the system during the time span $t-t_{0}$. We, moreover, assume that the system moves in the time independent potential $U(x)$. Hence, there are no external forces acting on the system and the non equilibrium state is entirely driven by the heat transfers from the reservoirs.

It follows from the structure of Fokker-Planck equation that the joint distribution 
$P\left(\mathbf{Q}, x t, x_{0} t_{0}\right)$ obeys the fundamental symmetry

$$
P\left(\mathbf{Q}, x t, x_{0} t_{0}\right)=\exp \left(-\beta_{k}\left(U(x)-U\left(x_{0}\right)\right)\right) \exp \left(-\sum_{n} \beta_{n k} Q_{n}\right) P\left(-\mathbf{Q}, x_{0} t, x t_{0}\right),
$$

as discussed in Sec. IIB. Here we have singled out the $\mathrm{k}$-th reservoir at temperature $T_{k}=$ $1 / \beta_{k}$ and, moreover, introduced the notation

$$
\beta_{n k}=\beta_{n}-\beta_{k}=1 / T_{n}-1 / T_{k}
$$

The symmetry (1.1) which is valid at all times is quite general for systems driven stochastically by heat reservoirs. The symmetry basically establishes a connection between the heat transfers during a transition from $x_{0}$ to $x$ and minus the heat transfers during the reverse transition from $x$ to $x_{0}$. The symmetry incorporates the general features of a reservoir-driven non equilibrium transition. As will become clear, the symmetry implies both the asymptotic fluctuation theorem for the cumulant generating function valid at long times, see e.g. [23, 24], the integral fluctuation theorem [19], and the exact fluctuation theorem proposed in [35] valid at all times.

In the present paper we discuss in detail the implications of the symmetry (1.1). For the purpose of a simple discussion we introduce in Sec. [1 the model of a single over damped particle moving in a time independent potential and at the same time driven by multiple heat reservoirs. This model allows for a simple derivation of the symmetry in (1.1). In Sec. III we consider the asymptotic long time regime and discuss the application of the symmetry to the cumulant generating function. Limiting the discussion to the harmonic potential we consider the branch cut structure of the cumulant generating function and the implications for the tails in the associated heat distribution. In Sec. IV we discuss implications of the symmetry valid at all times. We make contact with the trajectory formulation and associated entropy considerations. We also present a derivations of the integral fluctuation theorem and the exact fluctuation theorem. In Appendix E we summarise the extension of the formalism to the case of a time dependent potential modeling an external work protocol. This extension allows us to demonstrate the integral fluctuation theorem in the general case. In Sec. V we consider the case of a deterministic Hamiltonian system driven by heat reservoirs and present the corresponding symmetry for the joint distribution. In Sec. VI] we present a summery and a conclusion. Technical matters are deferred to a series of appendices. 


\section{SYSTEM COUPLED TO MULTIPLE HEAT RESERVOIRS - ONE DEGREE OF FREEDOM}

\section{A. General}

Here we consider for simplicity a single degree of freedom $x$ moving in the potential $U(x)$ and at the same time driven by multiple heat reservoirs maintained at temperatures $T_{n}=1 / \beta_{n}$. We restrict our discussion to the autonomous case where the potential is time independent. Associating the damping $\Gamma_{n}$ with the $\mathrm{n}$-th reservoir, the corresponding Langevin equation has the form, see e.g. [6, 19],

$$
\frac{d x(t)}{d t}=-\Gamma U^{\prime}(x)+\xi(t)
$$

where we have introduced the total damping, the total noise, and the noise correlations, i.e.,

$$
\begin{aligned}
& \Gamma=\sum_{n} \Gamma_{n}, \\
& \xi(t)=\sum_{n} \xi_{n}(t), \\
& \left\langle\xi_{n}(t) \xi_{m}\left(t^{\prime}\right)\right\rangle=2 \Gamma_{n} T_{n} \delta_{n m} \delta\left(t-t^{\prime}\right) ;
\end{aligned}
$$

here a prime denotes the spatial derivative $d / d x$. Correspondingly, the heat flux from the n-th reservoir is given by $F_{n}(x) U^{\prime}(x)$, where $F_{n}(x)=-\Gamma_{n} U^{\prime}(x)+\xi_{n}(t)$ is the force on the system originating from the n-th reservoir, i.e.,

$$
\frac{d Q_{n}(t)}{d t}=-\Gamma_{n}\left(U^{\prime}(x)\right)^{2}+U^{\prime}(x) \xi_{n}(t)
$$

The configuration is depicted in Fig. 1.

Since only a single degree of freedom is coupled to multiple reservoirs, the system is maintained in a state described by a Boltzmann-like probability distribution with an effective temperature. This is in contrast to for example a harmonic chain coupled to two reservoirs, where a genuine non equilibrium situation is established, see e.g. [34]. By inspection of the Langevin equation (2.1) for $x$ we infer the effective equilibrium temperature

$$
T=\frac{\sum_{n} \Gamma_{n} T_{n}}{\sum \Gamma_{n}},
$$

and the stationary distribution $P_{0}(x) \propto \exp (-U(x) / T)$. However, the individual heat fluxes between the reservoirs via the particle constitute a non equilibrium problem. From the 
equations of motion (2.1) and (2.5) we also infer

$$
\sum_{n} Q_{n}(t)=U(x)-U\left(x_{0}\right)
$$

expressing global energy conservation; here $x_{0}$ denotes the initial configuration. We assume in the following that $Q\left(t_{0}\right)=0$, i.e., we start sampling the heat at $t=t_{0}$. In other words, whereas the individual heat component $Q_{n}(t)$ is fluctuating, the sum $\sum_{n} Q_{n}(t)$ locks onto the nonfluctuating potential difference $U(x)-U\left(x_{0}\right)$.

The stochastic equations (2.1) and (2.5) define the problem we wish to study. However, for the present purposes it is more convenient to adhere to a Fokker-Planck description [36, 37]. Since the transfer of heat $\mathbf{Q}$ changes the state of the system and thus couples to the coordinate $x$, the distribution for the coordinate and heat is characterised by the joint distribution $P(\mathbf{Q}, x t)$ satisfying a Fokker-Planck equation with Liouville operator $L(\mathbf{Q}, x)$ [6, 37], i.e.,

$$
\begin{aligned}
& \frac{d P(\mathbf{Q}, x t)}{d t}=L(\mathbf{Q}, x) P(\mathbf{Q}, x t) \\
& L(\mathbf{Q}, x)=\sum_{n} \Gamma_{n}\left[T_{n} \frac{d^{2}}{d x^{2}}+\frac{d}{d x} U^{\prime}+\left(U^{\prime 2}+T_{n} U^{\prime \prime}\right) \frac{d}{d Q_{n}}+T_{n} U^{\prime 2} \frac{d^{2}}{d Q_{n}^{2}}+2 T_{n} U^{\prime} \frac{d^{2}}{d x d Q_{n}}\right]
\end{aligned}
$$

Since $L(\mathbf{Q}, x)$ is linear in $d / d Q_{n}$ it is convenient to introduce the characteristic function $C_{\boldsymbol{\lambda}}(x, t)$ defined according to

$$
C_{\boldsymbol{\lambda}}(x t)=\int \prod_{n} d Q_{n} \exp (\boldsymbol{\lambda} \mathbf{Q}) P(\mathbf{Q}, x t),
$$

where $\boldsymbol{\lambda} \mathbf{Q}=\sum_{n} \lambda_{n} Q_{n} . C_{\boldsymbol{\lambda}}(x, t)$ then satisfy the Fokker-Planck equation with Liouville operator below

$$
\begin{aligned}
& \frac{d C_{\boldsymbol{\lambda}}(x t)}{d t}=L_{\boldsymbol{\lambda}}(x) C_{\boldsymbol{\lambda}}(x t) \\
& L_{\boldsymbol{\lambda}}(x)=\sum_{n} \Gamma_{n}\left[T_{n} \frac{d^{2}}{d x^{2}}+U^{\prime}(x)\left(1-2 \lambda_{n} T_{n}\right) \frac{d}{d x}+\left(1-\lambda_{n} T_{n}\right)\left(U^{\prime \prime}(x)-\lambda_{n} U^{\prime 2}(x)\right)\right]
\end{aligned}
$$

We note that setting $\lambda_{n}=0$ corresponds to integrating over $Q_{n}$ and we have $C_{\boldsymbol{\lambda}=\mathbf{0}}(x t)=$ $P(x t)$, where $P(x t)$ is the distribution function for the coordinate $x$ in global equilibrium with the reservoirs at temperature $T$ given by (2.6). $P(x t)$ thus satisfies the Fokker-Planck equation $d P(x t) / d t=L_{\mathbf{0}}(x) P(x t)$, where $L_{\mathbf{0}}(x)=\Gamma\left(T d^{2} / d x^{2}+d / d x U^{\prime}(x)\right)$, with stationary distribution $P_{0}(x) \propto \exp (-U(x) / T)$. 


\section{B. Symmetry}

Incorporating the initial condition $x_{0}$ at time $t_{0}$ in the notation by setting $C_{\boldsymbol{\lambda}}(x t) \equiv$ $C_{\boldsymbol{\lambda}}\left(x t, x_{0} t_{0}\right)$, imposing the boundary condition $C_{\boldsymbol{\lambda}}\left(x t_{0}, x_{0} t_{0}\right)=\delta\left(x-x_{0}\right)$, and introducing the matrix notation

$$
L_{\boldsymbol{\lambda}}\left(x x^{\prime}\right)=L_{\boldsymbol{\lambda}}(x) \delta\left(x-x^{\prime}\right)
$$

the Fokker-Planck equation (2.11) takes the form

$$
\frac{d C_{\boldsymbol{\lambda}}\left(x t, x_{0} t_{0}\right)}{d t}=\int d x^{\prime} L_{\boldsymbol{\lambda}}\left(x x^{\prime}\right) C_{\boldsymbol{\lambda}}\left(x^{\prime} t, x_{0} t_{0}\right)
$$

In analogy with corresponding manipulations for the evolution operator in quantum mechanics [38], we obtain the formal solution

$$
C_{\boldsymbol{\lambda}}\left(x t, x_{0} t_{0}\right)=\left[\exp \left(L_{\boldsymbol{\lambda}}\left(t-t_{0}\right)\right)\right]\left(x, x_{0}\right)
$$

here the the $\mathrm{n}$-th term in the expansion of the exponential is defined according to $(1 / n !)(t-$ $\left.t_{0}\right)^{n} \int d x_{1} \cdots d x_{n-1} L_{\boldsymbol{\lambda}}\left(x, x_{1}\right) \cdots L_{\boldsymbol{\lambda}}\left(x_{n-1}, x_{0}\right)$.

Choosing a particular reservoir at temperature $T_{k}=1 / \beta_{k}$ inspection of the Liouville operator (2.12) in the form (2.13) yields the symmetry

$$
L_{\left\{\lambda_{n}\right\}}\left(x, x^{\prime}\right)=\exp \left(-\beta_{k}\left(U(x)-U\left(x^{\prime}\right)\right)\right) L_{\left\{\beta_{n k}-\lambda_{n}\right\}}\left(x^{\prime}, x\right),
$$

for details see Appendix B. From the solution (2.15) we then readily infer the corresponding symmetry for $C_{\left\{\lambda_{n}\right\}}$, i.e.,

$$
C_{\left\{\lambda_{n}\right\}}\left(x t, x_{0} t_{0}\right)=\exp \left(-\beta_{k}\left(U(x)-U\left(x_{0}\right)\right)\right) C_{\left\{\beta_{n k}-\lambda_{n}\right\}}\left(x_{0} t ; x t_{0}\right) .
$$

which holds for any choice of $k$. From the inverse Laplace transform (2.10) [39],

$$
P\left(\mathbf{Q}, x t, x_{0} t_{0}\right)=\int_{-i \infty}^{i \infty} \prod_{n} \frac{d \lambda_{n}}{2 \pi i} \exp (-\lambda \mathbf{Q}) C_{\boldsymbol{\lambda}}\left(x t, x_{0} t_{0}\right),
$$

we finally obtain (1.1), i.e.,

$$
P\left(\mathbf{Q}, x t, x_{0} t_{0}\right)=\exp \left(-\beta_{k}\left(U(x)-U\left(x_{0}\right)\right)\right) \exp \left(-\sum_{n} \beta_{n k} Q_{n}\right) P\left(-\mathbf{Q}, x_{0} t, x t_{0}\right),
$$


that again holds for all $k$. In the special case $\beta_{k}=0$ we obtain in particular the simpler symmetries

$$
\begin{aligned}
& C_{\left\{\lambda_{n}\right\}}\left(x t, x_{0} t_{0}\right)=C_{\left\{\beta_{n}-\lambda_{n}\right\}}\left(x_{0} t, x t_{0}\right), \\
& P\left(\mathbf{Q}, x t, x_{0} t_{0}\right)=\exp \left(-\sum_{n} \beta_{n} Q_{n}\right) P\left(-\mathbf{Q}, x_{0} t, x t_{0}\right) .
\end{aligned}
$$

This concludes the demonstration of the symmetries for the characteristic function and the joint heat distribution. In the next section we consider the implications for the cumulant generating function.

\section{CUMULANT GENERATING FUNCTION}

\section{A. General}

At long time the characteristic function $C_{\left\{\lambda_{n}\right\}}\left(x t, x_{0} t_{0}\right)$ is dominated by the largest eigenvalue $\mu_{0}(\boldsymbol{\lambda})$ of the Liouville operator $L_{\boldsymbol{\lambda}}$. This follows heuristically from the solution (2.15) and can be made more precise by considering the spectral representation of $L_{\boldsymbol{\lambda}}\left(x, x_{0}\right)$; this discussion is deferred to Appendix A. We have at long times compared to $t_{0}$

$$
C_{\boldsymbol{\lambda}}\left(x t, x_{0} t_{0}\right)=A_{0}\left(x x_{0}, \boldsymbol{\lambda}\right) \exp \left(\mu_{0}(\boldsymbol{\lambda}) t\right)
$$

where $\mu_{0}(\boldsymbol{\lambda})$ is the cumulant generating function. The prefactor $A_{0}\left(x x_{0}, \boldsymbol{\lambda}\right)$ is expressed in terms of the eigenfunctions associated with the eigenvalue $\mu_{0}(\boldsymbol{\lambda})$, for details see Appendix A. From the definition (2.10) we also have, denoting the constrained average by the subscript $x x_{0}$,

$$
\langle\exp (\boldsymbol{\lambda} \mathbf{Q}(t))\rangle_{x x_{0}}=A\left(x x_{0}, \boldsymbol{\lambda}\right) \exp \left(\mu_{0}(\boldsymbol{\lambda}) t\right)
$$

and it follows that $\mu_{0}$ yields the mean heat and higher cumulants, i.e. $\left\langle Q_{n}(t)\right\rangle_{x_{0}} / t=$ $A\left(x x_{0}, \mathbf{0}\right)\left(d \mu / d \lambda_{n}\right)_{\boldsymbol{\lambda}=0},\left(\left\langle Q(t)^{2}\right\rangle_{x x_{0}}-\left(\langle Q(t)\rangle_{x x_{0}}\right)^{2}\right) / t=A\left(x x_{0}, \mathbf{0}\right)\left(d^{2} \mu / d \lambda_{n}^{2}\right)_{\boldsymbol{\lambda}=0}$, etc.

Applying the symmetry (2.20) to (3.1) we readily obtain the usual asymptotic fluctuation theorem [23, 24] generalised to multiple reservoirs

$$
\mu_{0}\left(\left\{\lambda_{n}\right\}\right)=\mu_{0}\left(\left\{\beta_{n}-\lambda_{n}\right\}\right) .
$$

Likewise, we infer, applying the full symmetry (2.17) to (3.1), the generalised fluctuation theorem $\mu_{0}\left(\left\{\lambda_{n}\right\}\right)=\mu_{0}\left(\left\{\beta_{n k}-\lambda_{n}\right\}\right), \beta_{n k}=\beta_{n}-\beta_{k}$, for fixed $k$. Here we note that since 
$Q_{n}(t) \sim t$ the exponential $\exp \left(-\beta_{k}\left(U(x)-U\left(x_{0}\right)\right)\right.$ in (2.17) is subdominant in time compared with $\exp \left(\sum_{n}\left(\beta_{n k}-\lambda_{n}\right) Q_{n}(t)\right)$ at long times. Moreover, since this symmetry holds for any choice of $k$ we conclude that $\mu_{0}$ only depends on the difference variable

$$
\nu_{n m}=\lambda_{n}-\lambda_{m}
$$

and we have

$$
\mu_{0}\left(\left\{\nu_{n m}\right\}\right)=\mu_{0}\left(\left\{\beta_{n m}-\nu_{n m}\right\}\right)
$$

This relation generalises the usual asymptotic fluctuation theorem to the case of multiple reservoirs.

From (2.18) the heat distribution at long times is given by

$$
P\left(\mathbf{Q}, x t, x_{0} t_{0}\right)=\int_{-i \infty}^{i \infty} \prod_{n} \frac{d \lambda_{n}}{2 \pi i} A\left(x x_{0}, \boldsymbol{\lambda}\right) \exp \left(-\boldsymbol{\lambda} \mathbf{Q}+\mu_{0}(\boldsymbol{\lambda}) t\right) .
$$

Since the integral at long times is controlled by the exponential and $\mu_{0}$ depends on the difference $\lambda_{n}-\lambda_{m}$, it follows that the integral is invariant under the shift $\lambda_{n} \rightarrow \lambda_{n}+a$, where $a$ is arbitrary. Consequently, $\left.P\left(\mathbf{Q}, x t, x_{0} t_{0}\right)=\exp \left(-a \sum_{n} Q_{n}\right)\right) P\left(\mathbf{Q}, x t, x_{0} t_{0}\right)$ and we obtain the constraint

$$
\sum_{n} Q_{n}=0
$$

note that this constraint on the heat variables $\mathbf{Q}$ in the heat distribution $P\left(\mathbf{Q}, x t, x_{0} t_{0}\right)$ at long times is not in conflict with (2.7), i.e. $\sum_{n} Q_{n}(t)=U(x)-U\left(x_{0}\right)$ which expresses energy conservation for the fluctuating heat. At long times the dependence of $P$ on $Q_{n}$ is confined to the sub manifold determined by (3.7).

The condition (3.7) also follows from the symmetry (1.1) noting that $\exp \left(-\beta_{k}(U(x)-\right.$ $\left.\left.U\left(x_{0}\right)\right)\right)$ is subdominant in time and that $k$ can be chosen arbitrarily. We have in abbreviated notation

$$
P\left(Q_{1}, \cdots Q_{N}, t\right)=\delta\left(Q_{1}+\cdots+Q_{N}\right) P\left(Q_{1}, \cdots Q_{N-1}, t\right)
$$

In other words, for $N$ reservoirs there are only $N-1$ independent heat transfers as a result of the constraint in (3.7). For two reservoirs we have for example $P\left(Q_{1}, Q_{2}, t\right)=$ $\delta\left(Q_{1}+Q_{2}\right) P_{1}\left(Q_{1}, t\right)$, where $P_{1}(Q, t)$ is the heat distribution for reservoir 1 and we infer that 
$P_{2}(Q, t)=P_{1}(-Q, t)$, i.e., the distribution for the outgoing heat from reservoir 1 is identical to the distribution for incoming heat to reservoir 2, expressing conservation of energy.

By the usual steepest descent argument [24, 34, 40] the prefactor locks onto $A\left(x x_{0}, \boldsymbol{\lambda}^{*}\right)$, where $\lambda_{n}^{*}$ is a solution of $Q_{n} / t=\left(\partial \mu / \partial \lambda_{n}\right)_{\lambda_{n}=\lambda_{n}^{*}}$ and we have the scaling form

$$
\begin{aligned}
& P\left(\mathbf{Q}, x t, x_{0} t_{0}\right) \propto A\left(x x_{0}, \boldsymbol{\lambda}^{*}\right) \exp (-F(\mathbf{Q} / t) t), \\
& F(\mathbf{Q} / t)=-\mu_{0}\left(\boldsymbol{\lambda}^{*}\right)+\boldsymbol{\lambda}^{*} \mathbf{Q} / t
\end{aligned}
$$

where $F(\mathbf{q}), \mathbf{q}=\mathbf{Q} / t$, is the large deviation function in the case of multiple reservoirs. The symmetry (1.1) applied to $F(\mathbf{q})$ then yields the fluctuation theorem for the large deviation function

$$
F(\mathbf{q})=\sum_{n} \beta_{n k} q_{n}+F(-\mathbf{q}) \text { for arbitrary } k .
$$

\section{B. Branch points and tails}

It follows from general principles [40, 41] that the cumulant generating function $\mu_{0}(\boldsymbol{\lambda})$ is bounded and possesses a branch cut structure. For large $\mathbf{Q}$ the Laplace transform in (3.6), when closing the contour in its evaluation, samples the edges of the branch cuts closest to the origin determining the tails of the heat distribution. We note that in principle the singular structure of the prefactor $A\left(x x_{0}, \boldsymbol{\lambda}\right)$ can also influence the tails. This issue has been discussed in detail for the case of a particle driven by two heat reservoirs [42, 43]. In the present context we only analyse the contribution arising from the singular structure of $\mu_{0}(\boldsymbol{\lambda})$. In general $\mu_{0}(\boldsymbol{\lambda})$ is a downward convex function passing through the origin $\mu_{0}(\mathbf{0})=0$ due to normalzation. From the symmetry (3.5) it then also follows that $\mu_{0}\left(\left\{\beta_{n m}\right\}\right)=0$. Moreover, if $\mu_{0}$ has a branch point at $\nu_{n m}^{\mathrm{BP}}$ there will also be a branch point present at $\beta_{n m}-\nu_{n m}^{\mathrm{BP}}$.

In order to be more specific we return to the model in Sec. II and note that the similarity transformation $\exp (g)[6]$, where

$$
g(x, \boldsymbol{\lambda})=U(x) \frac{\sum_{n} \Gamma_{n} T_{n}\left(\lambda_{n}-\beta_{n} / 2\right)}{\Gamma T},
$$

maps the Liouville operator $L_{\boldsymbol{\lambda}}$ in (2.12) to a Hermitian Schrödinger form, i.e., $L_{\lambda}^{S}=$ $\exp (-g) L_{\boldsymbol{\lambda}} \exp (g)$, where

$$
L_{\boldsymbol{\lambda}}^{S}(x)=\Gamma\left[T \frac{d^{2}}{d x^{2}}+\frac{1}{2} U^{\prime \prime}-\frac{1}{4 T}\left(\frac{\omega}{\Gamma}\right)^{2} U^{\prime 2}\right] .
$$


Here

$$
\begin{aligned}
& \omega^{2}=\sum_{n m} \Gamma_{n} \Gamma_{m}\left[1-F_{n m}\right] \\
& F_{n m}=2 \nu_{n m}\left(T_{n}-T_{m}+T_{n} T_{m} \nu_{n m}\right) .
\end{aligned}
$$

Consulting Appendix $\mathrm{C}$ it follows that $L_{\boldsymbol{\lambda}}$ and $L_{\boldsymbol{\lambda}}^{S}$ have identical spectra, in particular the same largest eigenvalue $\mu_{0}$. From the structure of $L_{\lambda}^{S}$ it, moreover, follows that $\mu_{0}$ depends on the form of $U$ and parametrically on the temperature dependent parameter $\omega$. We also note that the function $F_{n m}$ is invariant under the transformation $\nu_{n m} \rightarrow \beta_{n m}-\nu_{n m}$, in accordance with (3.5)

In order to obtain an explicit expression for $\mu_{0}$ we consider in the following a harmonic oscillator potential $U(x)=x^{2} / 2$. Since $U^{\prime \prime}(x)=1$ and $U^{\prime}(x)=x$ the operator (3.13) corresponds to a quantum oscillator with mass $1 / 2 T$ moving in the potential $(1 / 4 T)(\omega / \Gamma)^{2} x^{2}-1 / 2$. Referring to Appendix D the discrete spectrum is given by $\mu_{n}\left(\left\{\nu_{n m}\right\}\right)=(1 / 2)(\Gamma-\omega)-\omega n, n=0,1, \cdots$, and we obtain in particular the cumulant generating function $\mu_{0}(\omega)=(1 / 2)(\Gamma-\omega)$. In more detail inserting (3.14)

$$
\mu_{0}\left(\left\{\nu_{n m}\right\}\right)=\frac{1}{2}\left(\Gamma-\left(\sum_{n m} \Gamma_{n} \Gamma_{m}\left[1-F_{n m}\right]\right)^{1 / 2}\right) .
$$

\section{One reservoir}

The situation is simple in the case of a single reservoir. We have $\lambda_{n}=\lambda$, i.e., $\nu_{n m}=0$ and from (3.14) $F_{n m}=0$. $\omega$ locks onto $\Gamma^{2}$ and we have $\mu_{0}=0$ for all $\lambda$, i.e., a vanishing cumulant generating function. This is consistent with the observation that a single degree of freedom coupled to a single heat reservoir is maintained in equilibrium at temperature $T$ with a bounded heat distribution [6, 33].

\section{Two reservoirs}

The case of two reservoirs with dampings $\Gamma_{1}$ and $\Gamma_{2}$ and temperatures $T_{1}$ and $T_{2}$ was considered by Derrida et al. [44], see also [33, 42]. Here we have $\nu=\lambda_{1}-\lambda_{2}, F_{12}=$ $2 \nu\left(T_{1}-T_{2}+T_{1} T_{2} \nu\right), \omega^{2}=\Gamma^{2}-2 \Gamma_{1} \Gamma_{2} F_{12}$, and the cumulant generating function is given by 
the explicit expression

$$
\mu_{0}(\nu)=\frac{1}{2}\left(\Gamma-\left(\Gamma^{2}+4 \Gamma_{1} T_{1} \Gamma_{2} T_{2} \nu\left(\beta_{12}-\nu\right)\right)^{1 / 2}\right) .
$$

The cumulant generating function is a downward convex function passing through $\mu_{0}(0)=0$ and $\mu_{0}\left(\beta_{12}\right)=0$. It, moreover, obeys the symmetry $\mu_{0}(\nu)=\mu_{0}\left(\beta_{12}-\nu\right)$. Closing off reservoir 2 by setting $\Gamma_{2}=0$ we have $\mu=0$ since a single particle driven by one reservoir is in equilibrium. The branch points are given by [33]

$$
\nu_{ \pm}=\frac{1}{2}\left(\beta_{12} \pm\left(\beta_{12}^{2}+\Gamma^{2} / \Gamma_{1} T_{1} \Gamma_{2} T_{2}\right)^{1 / 2}\right) .
$$

In the equal temperature case for $T_{1}=T_{2}=T$, i.e., $\beta_{12}=0$ we have in particular the branch points $\nu_{ \pm}= \pm(1 / 2 T)\left(\left(\Gamma_{1}+\Gamma_{2}\right) / \sqrt{\Gamma_{1} \Gamma_{2}}\right)$. For $\Gamma_{1}=\Gamma_{2}$ the branch points are independent of the damping and located at

$$
\nu_{ \pm}= \pm \frac{1}{T}
$$

\section{Multiple reservoirs}

For multiple reservoirs the analysis is based on the general expression in (3.16) originating from the harmonic potential case. We, moreover, for simplicity consider the case of $N$ reservoirs with identical damping constants, i.e., $\Gamma_{n}=\tilde{\Gamma}$ and thus $\Gamma=N \tilde{\Gamma}$. We find

$$
\mu_{0}\left(\left\{\nu_{n m}\right\}\right)=\frac{\tilde{\Gamma}}{2}\left(N-\left(\sum_{n m}\left[1-F_{n m}\right]\right)^{1 / 2}\right)
$$

and the branch point condition reads $\sum_{n m}\left[1-F_{n m}\right]=0$. This is still a complex expression to analyse; however, focussing on reservoir 1 by setting $\lambda_{1}=\lambda$ and $\lambda_{n}=0$, for $n \neq 1$ we have $\nu_{1 n}=\lambda$ for $n=2,3, \cdots, N$ and we obtain the branch points

$$
\nu_{ \pm}= \pm \frac{N}{2 \sqrt{N-1}} \frac{1}{T}
$$

For $N=2$ we recover (3.19); for $N=3$ we have $\nu_{ \pm}= \pm(1 / T) 3 / 2 \sqrt{2}$. As the number of reservoirs increase the branch points recede to infinity as $\sim \sqrt{N} / 2 T$. 


\section{Heat distribution}

For small $\lambda_{n}$ and assuming $\beta_{n m}$ small we can replace $\mu_{0}$ by a parabolic approximation consistent with the symmetry (3.5) and the boundary condition $\mu_{0}(\mathbf{0})=0$ i.e.,

$$
\mu_{0}\left(\left\{\nu_{n m}\right\}\right)=\sum_{n m} a_{n m} \nu_{n m}\left(\beta_{n m}-\nu_{n m}\right)
$$

Here $a_{n m}$ is symmetric and related to the mean heat flux according to $\left\langle q_{p}\right\rangle=2 \sum_{n} a_{p n} \beta_{p n}$. From the steepest descent calculation we then obtain after some algebra the following expression for the large deviation function

$$
F\left(\left\{\lambda_{n}\right\}\right)=\sum_{n m} a_{n m} \nu_{n m}^{*}\left(\beta_{n m}-\nu_{n m}^{*}\right)-\sum_{m} \lambda_{m}^{*} q_{m}
$$

where

$$
\begin{aligned}
& \lambda_{n}^{*}=\frac{1}{2} \sum_{p} K_{n p}\left(\sum_{m} a_{p m} \beta_{p m}-\frac{q_{p}}{2}\right), \\
& K_{n p}=\left(\delta_{n p} \sum_{q} a_{p q}-a_{n p}\right)^{-1} .
\end{aligned}
$$

Note that $F$ is quadratic in $q_{n}$ yielding a Gaussian heat distribution for small $q_{n}$; for the corresponding analysis in the case of two reservoirs, see [34].

Regarding the tails in the heat distribution the transform in (3.6) samples for large $q_{n}$ the branch points in $\mu_{0}$. Focussing on the heat distribution for reservoir 1 in the presence of the other $N-1$ reservoirs we thus obtain

$$
\begin{aligned}
& P\left(q_{1}\right) \sim \exp \left(-\nu_{+} q_{1} t\right) \text { for large positive } q_{1}, \\
& P\left(q_{1}\right) \sim \exp \left(-\left|\nu_{-}\right|\left|q_{1}\right| t\right) \text { for large negative } q_{1},
\end{aligned}
$$

where $\nu_{ \pm}$for $N$ reservoirs is given by (3.21).

\section{FLUCTUATION THEOREMS}

\section{A. Exact fluctuation theorem}

Here we discuss further consequences of the symmetry (1.1) valid at all times. Multiplying both sides with $\exp \left(-\beta_{k} U\left(x_{0}\right)\right)$, integrating over $x$ and $x_{0}$, and exchanging $x$ and $x_{0}$ in the 
integral on the left hand side, we obtain the identity

$$
P^{(k)}\left(\mathbf{Q}, t, t_{0}\right)=\exp \left(-\sum_{n} \beta_{n k} Q_{n}\right) P^{(k)}\left(-\mathbf{Q}, t, t_{0}\right)
$$

where we have defined the average

$$
P^{(k)}\left(\mathbf{Q}, t, t_{0}\right)=\int d x d x_{0} P\left(\mathbf{Q}, x_{0} t, x t_{0}\right) \exp \left(-\beta_{k} U\left(x_{0}\right)\right)
$$

Here $P^{(k)}\left(\mathbf{Q}, t, t_{0}\right)$ is the joint heat distribution associated with a non equilibrium transition from $x_{0}$ at time $t_{0}$ to $x$ at time $t$ with the system in equilibrium with the $k$-th reservoir at the initial time $t_{0}$. The expression (4.1) is a fluctuation theorem valid at all times and is a particular case of a more general expression first derived by Cuetara et al. [35] for a system in contact with several energy and particle reservoirs, on the basis of entropic considerations at the level of single trajectories.

In the case of two reservoirs setting $k=2$ we obtain in particular for the joint heat distribution $P^{(2)}\left(Q_{1}, Q_{2}, t, t_{0}\right)=\exp \left(-\left(\beta_{1}-\beta_{2}\right) Q_{1}\right) P^{(2)}\left(-Q_{1},-Q_{2}, t, t_{0}\right)$ or integrating over $Q_{2}$ the fluctuation theorem

$$
P^{(2)}\left(Q_{1}, t, t_{0}\right)=\exp \left(-\left(\beta_{1}-\beta_{2}\right) Q_{1}\right) P^{(2)}\left(-Q_{1}, t, t_{0}\right)
$$

The interpretation is straightforward, see [35]. Preparing the system in equilibrium at time $t_{0}$ with the reservoir at temperature $T_{2}$ and subsequently monitoring the heat transferred from the reservoir at temperature $T_{1}$, the heat distribution obeys the fluctuation theorem in (4.3) valid at all times.

In the case of multiple reservoirs we prepare the system in equilibrium with the k-th reservoir at temperature $T_{k}$ at time $t_{0}$ and the fluctuation theorem (4.1) applies to the joint heat distribution for the other reservoirs. Note that whereas the asymptotic fluctuation theorem (3.5) for the cumulant generating function is generic in the sense that it is independent of the potential $U(x)$, the fluctuation theorem in the present context requires that the system is in equilibrium with distribution $\exp \left(-\beta_{k} U\left(x_{0}\right)\right)$ with one of the reservoirs at the initial time. 


\section{B. Trajectory interpretation}

\section{Time independent potential - no work protocol}

In order to establish contact with the trajectory point of view pursued by Seifert [19], based on the stochastic thermodynamics scheme by Sekimoto [45], and the role of entropy changes in the course of the non equilibrium time evolution, we express the definition of the characteristic function in the form

$$
C_{\boldsymbol{\lambda}}\left(x t, x_{0} t_{0}\right)=\left\langle\exp \left(\sum_{n} \lambda_{n} Q_{n}(t)\right) \delta\left(x-f\left(t, x_{0} t_{0}\right)\right)\right\rangle .
$$

Here $f\left(t, x_{0} t_{0}\right)$ is a solution of the Langevin equation (2.1) for a specific noise realisation $\xi(t)$ defining a forward trajectory in configuration space from the initial configuration $x_{0}$ at time $t_{0}$ to the final configuration $x$ at time $t$. Likewise, solving $x=f\left(t, x_{0} t_{0}\right)$ for $x_{0}$, i.e.,

$x_{0}=\tilde{f}\left(t, x t_{0}\right)$, we identity a backward trajectory from $x$ at time $t_{0}$ to $x_{0}$ at time $t$. The symmetry (2.20) then reads

$$
\left\langle\exp \left(\sum_{n} \lambda_{n} Q_{n}(t)\right) \delta\left(x-f\left(t, x_{0} t_{0}\right)\right)\right\rangle=\left\langle\exp \left(\sum_{n}\left(\beta_{n}-\lambda_{n}\right) Q_{n}(t)\right) \delta\left(x_{0}-\tilde{f}\left(t, x t_{0}\right)\right)\right\rangle .(4 .
$$

For a particular noise realisation defining a trajectory in configuration space we obtain

$$
\exp \left(\sum_{n} \lambda_{n} Q_{n}^{\mathrm{F}}(t)\right) \delta\left(x-f\left(t, x_{0} t_{0}\right)\right)=\exp \left(\sum_{n}\left(\beta_{n}-\lambda_{n}\right) Q_{n}^{\mathrm{B}}(t)\right) \delta\left(x_{0}-\tilde{f}\left(t, x t_{0}\right)\right) .
$$

Note that $Q_{n}^{\mathrm{F}}(t)$ is a solution of the Langevin equation (2.5). Since $\delta\left(x-f\left(t, x_{0} t_{0}\right)\right)$ defines a forward trajectory the heat transfer $Q_{n}^{\mathrm{F}}(t)$ is associated with this trajectory; likewise, $Q_{n}^{\mathrm{B}}(t)$ is associated with a backward trajectory. For the special choice $\lambda_{n}=\beta_{n}$ we obtain in particular

$$
\exp \left(\sum_{n} \beta_{n} Q_{n}^{\mathrm{F}}(t)\right) \delta\left(x-f\left(t, x_{0} t_{0}\right)\right)=\delta\left(x_{0}-\tilde{f}\left(t, x t_{0}\right)\right)
$$

This relationship is completely equivalent to the analysis in [19] based on the path integral formulation of the solution to the Fokker-Planck for the distribution $P\left(x t, x_{0} t_{0}\right)$.

Setting $\lambda_{n}=\beta_{n}$ in (4.5) and integrating over the initial position $x_{0}$ we obtain

$$
\int d x_{0}\left\langle\exp \left(\sum_{n} \beta_{n} Q_{n}(t)\right) \delta\left(x-f\left(t, x_{0} t_{0}\right)\right)\right\rangle=1,
$$


where we have used the normalisation condition $\int d x_{0}\left\langle\delta\left(x_{0}-\tilde{f}\left(t, x t_{0}\right)\right)\right\rangle=1$ or, equivalently, $\int d x_{0} P\left(x_{0} t, x t_{0}\right)=1$, i.e., the conservation of probability.

Introducing the total increase of the heat bath entropy

$$
S_{\text {bath }}(t)=-\sum_{n} \beta_{n} Q_{n}(t)
$$

we can also express (4.8) in the form of a fluctuation theorem for the bath entropy

$$
\int d x_{0}\left\langle\exp \left(-S_{\text {bath }}(t)\right)\right\rangle_{x t, x_{0} t_{0}}=1
$$

This fluctuation theorem states that integrated over the initial configuration $x_{0}$ with weight one, the constrained average of $\exp \left(-S_{\text {bath }}(t)\right)$ along forward trajectories equals unity. The fluctuation theorem relates to the total reservoir entropy production in the course of a non equilibrium transition. The fluctuation theorem is a consequence of a basic symmetry of the joint heat-position distribution combined with the normalisation of the distribution for the position. We also note that the joint distribution incorporates on the Fokker-Planck level the stochastic thermodynamics scheme developed within a Langevin formulation [19, 45].

\section{Time dependent potential - with work protocol}

In the case of a work protocol characterised by a time dependent potential $U(x t)$ modelling work performed on the system, the symmetries in (2.17) and (2.20) are not available since the Liouville operator acquires an explicit time dependence. The extension of the scheme to the time dependent case is summarised in Appendix E. Whereas the fundamental symmetry still applies to the Liouville operator, i.e.,

$$
L_{\left\{\lambda_{n}\right\}}\left(x x^{\prime}, t\right)=\exp \left(-\beta_{k}\left(U(x t)-U\left(x^{\prime} t\right)\right)\right) L_{\left\{\beta_{n k}-\lambda_{n}\right\}}\left(x^{\prime} x, t\right)
$$

the characteristic function is given by the time ordered product [38],

$$
C_{\boldsymbol{\lambda}}\left(x t, x^{\prime} t^{\prime}\right)=T\left[\exp \left(\int_{t^{\prime}}^{t} d t^{\prime \prime} \hat{L}_{\boldsymbol{\lambda}}\left(t^{\prime \prime}\right)\right)\right]\left(x x^{\prime}\right) .
$$

Considering for example the second order term in the expansion we obtain applying the symmetry

$$
\begin{aligned}
& C_{\left\{\lambda_{n}\right\}}^{(2)}\left(x t, x^{\prime} t^{\prime}\right)=\int_{t^{\prime}}^{t} d t_{1} \int_{t^{\prime}}^{t_{1}} d t_{2} \int d y L_{\left\{\lambda_{n}\right\}}\left(x y, t_{1}\right) L_{\left\{\lambda_{n}\right\}}\left(y x^{\prime}, t_{2}\right)= \\
& \left.\int_{t^{\prime}}^{t} d t_{1} \int_{t^{\prime}}^{t_{1}} d t_{2} \int d y L_{\left\{\beta_{n k}-\lambda_{n}\right\}} y x, t_{1}\right) L_{\left\{\beta_{n k}-\lambda_{n}\right\}}\left(x^{\prime} y, t_{2}\right) e^{-\beta_{k}\left(U\left(x t_{1}\right)-U\left(y t_{1}\right)+U\left(y t_{2}\right)-U\left(x^{\prime} t_{2}\right)\right.},
\end{aligned}
$$


and we note that the time dependent protocol as exemplified by the time dependence of $U$ becomes entirely entangled in the time integrations. On the contrary, in the time independent case the factors $\exp \left(-\beta_{k} U(x)\right)$ in the expansion of the exponential in (4.12) cancel and we recover the symmetry in (2.17).

We also note in passing that in the case of a moving harmonic potential of the form $U(x t) \propto(x-v t)^{2}$, as discussed in [6], the time ordered expression reduces to integration over Gaussians (yielding error functions) and can possible be reduced. However, we abstain from such an analysis in the present context; we refer to [6] for a steepest descent analysis.

With respect to the bath-fluctuation theorem (4.10) we note that setting $\beta_{k}=0$ we have in operator form, see Appendix E,

$$
C_{\left\{\lambda_{n}\right\}}\left(x t, x^{\prime} t^{\prime}\right)=T\left[\exp \left(\int_{t^{\prime}}^{t} d t^{\prime \prime} \hat{L}_{\left\{\beta_{n}-\lambda_{n}\right\}}^{*}\left(t^{\prime \prime}\right)\right)\right]\left(x x^{\prime}\right),
$$

and in particular setting $\beta_{n}=\lambda_{n}$

$$
C_{\left\{\beta_{n}\right\}}\left(x t, x^{\prime} t^{\prime}\right)=T\left[\exp \left(\int_{t^{\prime}}^{t} d t^{\prime \prime} \hat{L}_{\{0\}}^{*}\left(t^{\prime \prime}\right)\right)\right]\left(x x^{\prime}\right) .
$$

It follows from the form of the Liouville operator in (6.30) that $\int d x L_{\{0\}}\left(x x^{\prime}, t\right)=$ 0 , expressing conservation of probability in the Fokker-Planck equation for $P\left(x t, x^{\prime} t^{\prime}\right)$, $d P\left(x t, x^{\prime} t^{\prime}\right) / d t=\int d x^{\prime \prime} L_{\{0\}}\left(x x^{\prime \prime}, t\right) P\left(x^{\prime \prime} t, x^{\prime} t^{\prime}\right)$. Correspondingly, for the transposed Liouvillian $L^{*}$ we have $\int d x^{\prime} L_{\{0\}}^{*}\left(x x^{\prime}, t\right)=0$ and it follows from (4.15) that also in the time dependent case do we obtain (4.8), yielding the fluctuation theorem (4.10).

The above analysis might appear cumbersome and possibly superfluous. General physical arguments imply that the fluctuation theorem for the bath entropy only monitors the transfer of heat and thus does not depend on the applied work protocol.

\section{Integral fluctuation theorem}

Seifert has proposed an integral fluctuation theorem valid at all times based on entropy production and consumption on the trajectory level [19]. Within the present context the integral fluctuation theorem follows readily from (4.10) and is therefore a consequence of the basic symmetry of the Liouville operator.

The bath fluctuation theorem was formulated as an integral condition for the constrained average of the fluctuating heat or entropy $-S_{\text {bath }}(t)$ for an initial configuration at $x_{0}$ at time 
$t_{0}$ and a final configuration $x$ at time $t$. In order to make contact with the integral fluctuation theorem one must consider fluctuating initial and final configuration characterised by the normalised distributions $P_{t}(x)$ and $P_{0}\left(x_{0}\right)$.

Based on the path integral formulation of the distribution $P\left(x t, x^{\prime} t^{\prime}\right)$ it was shown in [19] that one can define entropy production and consumption for an individual trajectory from $x_{0}$ at time $t_{0}$ to $x$ at time $t$ based on the Gibbs expression $S=-\sum_{n} p_{n} \ln p_{n}$. By inspection of the entropy associated with the forward and backward trajectories one easily extracts the entropy associated with the contact with the heat baths, $S_{\text {bath }}$. The additional entropy associated with the non equilibrium transition itself on the trajectory level is then given in terms of the initial and final distribution as $S_{\text {sys }}=\ln P_{0}\left(x_{0}\right)-\ln P_{t}(x)$.

Averaging over the initial distribution $P_{0}\left(x_{0}\right)$ we infer from (4.10)

$$
\int d x_{0}\left\langle\exp \left(-S_{\text {bath }}(t)-\ln P_{0}\left(x_{0}\right)\right)\right\rangle_{x t, x_{0} t_{0}} P_{0}\left(x_{0}\right)=1
$$

where the distribution $P_{0}\left(x_{0}\right)$ is balanced by the entropy contribution $\ln P_{0}\left(x_{0}\right)$. Finally, integrating over the final state $x$ with the normalised weight $P_{t}(x)$, i.e., $\int d x P_{t}(x)=1$, we obtain the integral fluctuation theorem proposed by Seifert [19]

$$
\int d x \int d x_{0}\left\langle\exp \left(-S_{\text {total }}(t)\right)\right\rangle_{x t, x_{0} t_{0}} P_{0}\left(x_{0}\right)=1
$$

or

$$
\left\langle\exp \left(-S_{\text {total }}(t)\right)\right\rangle=1
$$

where the total entropy is given by

$$
\begin{aligned}
& S_{\text {total }}=S_{\text {bath }}(t)+S_{\text {sys }}, \\
& S_{\text {sys }}=\ln P_{0}\left(x_{0}\right)-\ln P_{t}(x) .
\end{aligned}
$$

\section{RESERVOIR-DRIVEN HAMILTONIAN SYSTEM}

The last issue to be considered in this section is the case of a system with many degrees of freedom. We consider an over damped system coupled to multiple reservoirs described by 
the Hamiltonian $H(\mathbf{x p})$. For the equations of motion for $\mathbf{x}, \mathbf{p}$ and $\mathbf{Q}$ we obtain

$$
\begin{aligned}
& \frac{d x_{n}}{d t}=p_{n}, \\
& \frac{d p_{n}}{d t}=-\frac{d H}{d x_{n}}-\Gamma_{n} p_{n}+\xi_{n}, \\
& \left\langle\xi_{n} \xi_{m}\right\rangle(t)=2 \Gamma_{n} T_{n} \delta_{n m} \delta(t), \\
& \frac{d Q_{n}}{d t}=p_{n}\left(-\Gamma_{n} p_{n}+\xi_{n}\right),
\end{aligned}
$$

and the conservation law

$$
\sum_{n} Q_{n}(t)=H(\mathbf{x p})-H\left(\mathbf{x}_{0} \mathbf{p}_{0}\right)
$$

where $\left(\mathbf{x}_{0}, \mathbf{p}_{0}\right)$ is the initial phase space point at time $t_{0}$ and $(\mathbf{x}, \mathbf{p})$ the phase space point at time $t$.

The Fokker-Planck equation for the joint distribution $P(\mathbf{Q}, \mathbf{x p} t)$ has the form

$$
\begin{aligned}
\frac{d P(\mathbf{Q}, \mathbf{x p} t)}{d t} & =\left(L_{0}(\mathbf{x p})+L_{\mathbf{Q}}(\mathbf{p})\right) P(\mathbf{Q}, \mathbf{x p} t) \\
L_{0}(\mathbf{x p}) & =\sum_{n}\left[\left(\frac{d H}{d x_{n}} \frac{d}{d p_{n}}-\frac{d H}{d p_{n}} \frac{d}{d x_{n}}\right)+\Gamma_{n}\left(T_{n} \frac{d^{2}}{d p_{n}^{2}}+\frac{d}{d p_{n}} p_{n}\right)\right] \\
L_{\mathbf{Q}}(\mathbf{p}) & =\sum_{n} \Gamma_{n}\left[T_{n} p_{n}^{2} \frac{d^{2}}{d Q_{n}^{2}}+2 T_{n} p_{n} \frac{d^{2}}{d Q_{n} d p_{n}}+\left(p_{n}^{2}+T_{n}\right) \frac{d}{d Q_{n}}\right]
\end{aligned}
$$

we note the appearance of a Poisson bracket accounting for the deterministic dynamics. Likewise, the characteristic function

$$
C_{\boldsymbol{\lambda}}(\mathbf{x p} t)=\int \prod_{n} d Q_{n} \exp (\boldsymbol{\lambda} \mathbf{Q}) P(\mathbf{Q}, \mathbf{x p} t)
$$

is governed by the corresponding Fokker-Planck equation

$$
\begin{aligned}
\frac{d C_{\boldsymbol{\lambda}}(\mathbf{x p} t)}{d t}= & L_{\boldsymbol{\lambda}}(\mathbf{x p}) C_{\boldsymbol{\lambda}}(\mathbf{x p}, t) \\
L_{\boldsymbol{\lambda}}(\mathbf{x p})= & \sum_{n}\left(\frac{d H}{d x_{n}} \frac{d}{d p_{n}}-\frac{d H}{d p_{n}} \frac{d}{d x_{n}}\right) \\
& +\sum_{n} \Gamma_{n}\left[T_{n} \frac{d^{2}}{d p_{n}^{2}}+\left(1-2 \lambda_{n} T_{n}\right) p_{n} \frac{d}{d p_{n}}+\left(1-\lambda_{n} T_{n}\right)\left(1-\lambda_{n} p_{n}^{2}\right)\right] .
\end{aligned}
$$

Setting $L_{\boldsymbol{\lambda}}\left(\mathbf{x p}, \mathbf{x}^{\prime} \mathbf{p}^{\prime}\right)=L_{\boldsymbol{\lambda}}(\mathbf{x p}) \delta\left(\mathbf{x}-\mathbf{x}^{\prime}\right) \delta\left(\mathbf{p}-\mathbf{p}^{\prime}\right)$ and including a time reversal operation applied to the Poisson bracket in accordance with microscopic reversibility, we note that the 
momentum as a result will change sign, i.e., $\mathbf{p} \rightarrow-\mathbf{p}$. By inspection we find the fundamental symmetry analogous to (2.16),

$$
L_{\left\{\lambda_{n}\right\}}\left(\mathbf{x p} ; \mathbf{x}^{\prime} \mathbf{p}^{\prime}\right)=\exp \left(-\beta_{k}\left(H(\mathbf{x p})-H\left(\mathbf{x}^{\prime} \mathbf{p}^{\prime}\right)\right)\right) L_{\left\{\beta_{n k}-\lambda_{n}\right\}}\left(\mathbf{x}^{\prime}\left(-\mathbf{p}^{\prime}\right) ; \mathbf{x}(-\mathbf{p})\right),
$$

and from the spectral representation for the characteristic function, see Appendix A,

$$
C_{\left\{\lambda_{n}\right\}}\left(\mathbf{x p} t ; \mathbf{x}_{0} \mathbf{p}_{0}, t_{0}\right)=\exp \left(-\beta_{k}\left(H(\mathbf{x p})-H\left(\mathbf{x}_{0} \mathbf{p}_{0}\right)\right)\right) C_{\left\{\beta_{n k}-\lambda_{n}\right\}}\left(\mathbf{x}_{0}\left(-\mathbf{p}_{0}\right) t ; \mathbf{x}(-\mathbf{p}) t_{0}\right)
$$

From the inverse transform of (5.9)

$$
P\left(\mathbf{Q}, \mathbf{x p} t, \mathbf{x}_{0} \mathbf{p}_{0} t_{0}\right)=\int_{-i \infty}^{+i \infty} \prod_{n} \frac{d \lambda_{n}}{2 \pi i} \exp (-\lambda \mathbf{Q}) C_{\boldsymbol{\lambda}}\left(\mathbf{x p} t, \mathbf{x}_{0} \mathbf{p}_{0} t_{0}\right)
$$

we readily infer the symmetry

$$
\begin{aligned}
P\left(\mathbf{Q}, \mathbf{x p} t, \mathbf{x}_{0} \mathbf{p}_{0} t_{0}\right)= & \exp \left(-\beta_{k}\left(H(\mathbf{x p})-H\left(\mathbf{x}_{0} \mathbf{p}_{0}\right)\right)\right) \times \\
& \exp \left(-\sum_{n} \beta_{n k} Q_{n}\right) P\left(-\mathbf{Q}, \mathbf{x}_{0}\left(-\mathbf{p}_{0}\right) t, \mathbf{x}(-\mathbf{p}) t\right) .
\end{aligned}
$$

We conclude that the previous analysis in the case of a single degree of freedom can be carried over unchanged to the case of a Hamiltonian system with many degrees of freedom.

\section{SUMMARY AND CONCLUSION}

In the present paper we have considered general properties of systems driven by multiple heat reservoirs. The discussion has been based on the joint distribution for the heat transfers and system coordinates. Analysing the Fokker-Planck equation for the joint distribution we have identified a fundamental symmetry which relate the positive heat transfers associated with the non equilibrium progression of the system from an initial state to final state to the negative heat transfers associated with the reverse transition from the final state to the initial state. This symmetry which is specific to the multi reservoir case permits i) a generalisation of the asymptotic long time fluctuation theorem for the large deviation function or, equivalently, the cumulant generating function and yields corrections to the tails in the heat distributions, ii) a derivation of a recent exact fluctuation theorem for systems initially in equilibrium with a reservoir. Extending the analysis to the time dependent case we have also presented iii) a derivation of the integral fluctuation theorem. For simplicity

the main analysis is based on a model with one degree of freedom but also holds unaltered for systems with many degrees of freedom. 


\section{Appendices}

\section{A. Spectral representations}

The spectral representations of $L_{\boldsymbol{\lambda}}$ and $C_{\boldsymbol{\lambda}}$ incorporate in a convenient form the properties

of the Fokker-Planck equation (2.11)-(2.12) [36, 37]. Assuming a non degenerate spectrum and introducing a bi-orthogonal set, for details see [36], i.e., an orthonormal and complete set of left and right eigenstates, according to the eigenvalue equations

$$
\begin{aligned}
& \int d x^{\prime} L_{\boldsymbol{\lambda}}\left(x, x^{\prime}\right) \Psi_{n}^{R}\left(x^{\prime}, \boldsymbol{\lambda}\right)=\mu_{n}(\boldsymbol{\lambda}) \Psi_{n}^{R}(x, \boldsymbol{\lambda}), \\
& \int d x^{\prime} \Psi_{n}^{L}\left(x^{\prime}, \lambda\right) L_{\lambda}\left(x^{\prime}, x\right)=\mu_{n}(\lambda) \Psi_{n}^{L}(x, \lambda),
\end{aligned}
$$

with completeness and orthogonality properties

$$
\begin{aligned}
& \sum_{n} \Psi_{n}^{R}(x, \boldsymbol{\lambda}) \Psi_{n}^{L}\left(x^{\prime}, \boldsymbol{\lambda}\right)=\delta\left(x-x^{\prime}\right), \\
& \int d x \Psi_{n}^{R}(x, \boldsymbol{\lambda}) \Psi_{m}^{L}(x, \boldsymbol{\lambda})=\delta_{n m} .
\end{aligned}
$$

Thus, setting $C_{\boldsymbol{\lambda}}\left(x t, x_{0} t_{0}\right)=C_{\boldsymbol{\lambda}}\left(x, t-t_{0}\right) \delta\left(x-x^{\prime}\right)$, we obtain the spectral forms

$$
\begin{aligned}
& L_{\boldsymbol{\lambda}}\left(x, x^{\prime}\right)=\sum_{n} \mu_{n}(\boldsymbol{\lambda}) \Psi_{n}^{R}(x, \boldsymbol{\lambda}) \Psi_{n}^{L}\left(x^{\prime}, \boldsymbol{\lambda}\right), \\
& C_{\boldsymbol{\lambda}}\left(x t, x_{0} t_{0}\right)=\sum_{n} \exp \left(\mu_{n}(\boldsymbol{\lambda})\left(t-t_{0}\right)\right) \Psi_{n}^{R}(x, \lambda) \Psi_{n}^{L}\left(x_{0}, \boldsymbol{\lambda}\right) .
\end{aligned}
$$

We assume that the eigenvalue spectrum $\left\{\mu_{n}(\boldsymbol{\lambda})\right\}$ forms a decreasing sequence with largest eigenvalue $\mu_{0}(\boldsymbol{\lambda})$. Moreover, completeness yields the following boundary condition $C_{\boldsymbol{\lambda}}\left(x t, x_{0} t_{0}\right)=\delta\left(x-x_{0}\right)$ for $t=t_{0}$.

Applying the symmetry (2.17) to the spectral form (6.5) we find

$$
\begin{aligned}
& \mu_{n}\left(\left\{\nu_{n m}\right\}\right)=\mu_{n}\left(\left\{\beta_{n m}-\nu_{n m}\right\}\right), \\
& \Psi_{n}^{R}\left(x,\left\{\lambda_{n}\right\}\right)=\exp \left(-\beta_{k} U(x)\right) \Psi_{n}^{L}\left(x,\left\{\beta_{n k}-\lambda_{n}\right\}\right) .
\end{aligned}
$$

From the leading eigenvalue $\mu_{0}$ we thus obtain the generalisation (3.5) of the usual asymptotic fluctuation theorem. We also infer the prefactor $A_{n}\left(x x_{0}, \boldsymbol{\lambda}\right)=\Psi_{n}^{R}(x, \boldsymbol{\lambda}) \Psi_{n}^{L}\left(x_{0}, \boldsymbol{\lambda}\right)$. 


\section{B. The symmetry}

In order to demonstrate the symmetry (2.16), i.e.,

$$
L_{\left\{\lambda_{n}\right\}}\left(x, x^{\prime}\right)=\exp \left(-\beta_{k}\left(U(x)-U\left(x^{\prime}\right)\right)\right) L_{\left\{\beta_{n k}-\lambda_{n}\right\}}\left(x^{\prime}, x\right),
$$

we analyse in more detail the Liouville operator

$$
L_{\boldsymbol{\lambda}}\left(x, x^{\prime}\right)=\sum_{n} \Gamma_{n}\left[T_{n} \frac{d^{2}}{d x^{2}}+U^{\prime}\left(1-2 \lambda_{n} T_{n}\right) \frac{d}{d x}+\left(1-\lambda_{n} T_{n}\right)\left(U^{\prime \prime}-\lambda_{n} U^{\prime 2}\right)\right] \delta\left(x-x^{\prime}\right) .
$$

Using the identities

$$
\begin{aligned}
e^{\beta_{k} U(x)} \frac{d}{d x} \delta\left(x-x^{\prime}\right) e^{-\beta_{k} U\left(x^{\prime}\right)} & =\left(\frac{d}{d x}-\beta_{k} U^{\prime}(x)\right) \delta\left(x-x^{\prime}\right), \\
e^{\beta_{k} U(x)} \frac{d^{2}}{d x^{2}} \delta\left(x-x^{\prime}\right) e^{-\beta_{k} U\left(x^{\prime}\right)} & =\left(\frac{d}{d x}-\beta_{k} U^{\prime}(x)\right)\left(\frac{d}{d x}-\beta_{k} U^{\prime}(x)\right) \delta\left(x-x^{\prime}\right), \\
U^{\prime}(x) \frac{d}{d x} \delta\left(x-x^{\prime}\right) & =-\left(U^{\prime}\left(x^{\prime}\right) \frac{d}{d x^{\prime}}-U^{\prime \prime}\left(x^{\prime}\right)\right) \delta\left(x-x^{\prime}\right),
\end{aligned}
$$

and setting $\lambda_{n} \rightarrow \beta_{n k}-\lambda_{n}$ it is easy to show that

$$
e^{\beta_{k} U(x)} L_{\left\{\lambda_{n}\right\}}\left(x, x^{\prime}\right) e^{-\beta_{k} U\left(x^{\prime}\right)}=L_{\left\{\beta_{n k}-\lambda_{n}\right\}}\left(x^{\prime}, x\right),
$$

and the symmetry (6.9) follows.

\section{Mapping to Schrödinger case}

The similarity transformation

$$
g(x, \boldsymbol{\lambda})=U(x) \frac{\sum_{n} \Gamma_{n} T_{n}\left(\lambda_{n}-\beta_{n} / 2\right)}{\Gamma T},
$$

maps the Liouville operator $L_{\boldsymbol{\lambda}}\left(x, x^{\prime}\right)$ in (2.12) to the Hermitian Schrödinger form $L_{\boldsymbol{\lambda}}^{S}\left(x, x^{\prime}\right)$, i.e.,

$$
L_{\boldsymbol{\lambda}}^{S}\left(x, x^{\prime}\right)=\exp (-g(x)) L_{\boldsymbol{\lambda}}\left(x, x^{\prime}\right) \exp \left(g\left(x^{\prime}\right)\right)
$$

where

$$
L_{\boldsymbol{\lambda}}^{S}(x)=\Gamma\left[T \frac{d^{2}}{d x^{2}}+\frac{1}{2} U^{\prime \prime}-\frac{1}{4 T}\left(\frac{\omega}{\Gamma}\right)^{2} U^{\prime 2}\right]
$$

The associated eigenvalue problem is

$$
\int d x^{\prime} L_{\boldsymbol{\lambda}}^{S}\left(x, x^{\prime}\right) \Psi_{n}\left(x^{\prime}, \boldsymbol{\lambda}\right)=\mu_{n}(\boldsymbol{\lambda}) \Psi_{n}(x, \boldsymbol{\lambda})
$$


with complete and orthogonal eigenstates,

$$
\begin{aligned}
& \sum_{n} \Psi_{n}(x, \boldsymbol{\lambda}) \Psi_{n}\left(x^{\prime}, \boldsymbol{\lambda}\right)=\delta\left(x-x^{\prime}\right) \\
& \int d x \Psi_{n}(x, \boldsymbol{\lambda}) \Psi_{m}(x, \boldsymbol{\lambda})=\delta_{n m}
\end{aligned}
$$

The spectral representation of $L_{\boldsymbol{\lambda}}\left(x, x^{\prime}\right)^{S}$ has the form

$$
L_{\boldsymbol{\lambda}}^{S}\left(x, x^{\prime}\right)=\sum_{n} \mu_{n}(\boldsymbol{\lambda}) \Psi_{n}(x, \boldsymbol{\lambda}) \Psi_{n}\left(x^{\prime}, \boldsymbol{\lambda}\right)
$$

We conclude that the eigenvalues $\mu(\boldsymbol{\lambda})$ of $L_{\boldsymbol{\lambda}}$ and $L_{\boldsymbol{\lambda}}^{S}$ are identical, whereas the eigenstates are transformed according to

$$
\begin{aligned}
& \Psi_{n}^{R}(x, \boldsymbol{\lambda})=\exp (g(x, \boldsymbol{\lambda})) \Psi_{n}(x, \boldsymbol{\lambda}), \\
& \Psi_{n}^{L}(x, \boldsymbol{\lambda})=\exp (-g(x, \boldsymbol{\lambda})) \Psi_{n}(x, \boldsymbol{\lambda}) .
\end{aligned}
$$

We also note that the identity $g\left(x,\left\{\lambda_{n}\right\}\right)+g\left(x,\left\{\beta_{n k}-\lambda_{n}\right\}\right)=\beta_{k} U$ ensures consistency with the symmetry (6.8).

\section{Harmonic potential}

For a harmonic potential $U(x)=x^{2} / 2$ the Schrödinger operator takes the form

$$
L_{\boldsymbol{\lambda}}^{S}(x)=\Gamma\left[T \frac{d^{2}}{d x^{2}}+\frac{1}{2}-\frac{1}{4 T}\left(\frac{\omega}{\Gamma}\right)^{2} x^{2}\right],
$$

corresponding to a quantum mechanical particle in the potential $(1 / 4 T)(\omega / \Gamma)^{2} x^{2}-1 / 2$. The spectrum and eigenstates are given by [46]

$$
\begin{aligned}
& \mu_{n}(\boldsymbol{\lambda})=\frac{1}{2}(\Gamma-\omega(\boldsymbol{\lambda}))-\omega(\boldsymbol{\lambda}) n \\
& \Psi_{n}(x)=\left(\frac{\omega}{2 \pi \Gamma T}\right)^{1 / 4}\left(\frac{1}{2^{n} n !}\right)^{1 / 2} \exp \left(-\frac{\omega x^{2}}{4 \Gamma T}\right) H_{n}\left(x \sqrt{\frac{\omega}{2 \Gamma T}}\right),
\end{aligned}
$$

where $H_{n}$ is the Hermite polynomial and $n=0,1,2, \cdots$.

\section{E. Time dependent potential}

Here we extend our discussion of a particle in a time independent potential driven by multiple reservoirs to the case where the system is subject to a time dependent protocol 
modeled here by a potential $U(x t)$ depending parametrically on $t$. More precisely, denoting the chosen protocol by $\lambda(t)$ we have set $U(x, \lambda(t)) \equiv U(x t)$. The equations of motion (2.1) and (2.5) then take the form

$$
\begin{aligned}
& \frac{d x(t)}{d t}=-\Gamma U^{\prime}(x t)+\xi(t) \\
& \frac{d Q_{n}(t)}{d t}=-\Gamma_{n}\left(U^{\prime}(x t)\right)^{2}+U^{\prime}(x t) \xi_{n}(t) .
\end{aligned}
$$

Correspondingly, the Fokker-Planck equation for the characteristic function $C_{\boldsymbol{\lambda}}(x, t)$ is

$$
\frac{d C_{\boldsymbol{\lambda}}(x t)}{d t}=L_{\boldsymbol{\lambda}}(x t) C_{\boldsymbol{\lambda}}(x t)
$$

where

$$
\begin{aligned}
L_{\boldsymbol{\lambda}}(x t)=\sum_{n} \Gamma_{n}[ & T_{n} \frac{d^{2}}{d x^{2}}+U^{\prime}(x t)\left(1-2 \lambda_{n} T_{n}\right) \frac{d}{d x}+ \\
& \left.+\left(1-\lambda_{n} T_{n}\right)\left(U^{\prime \prime}(x t)-\lambda_{n} U^{\prime 2}(x t)\right)\right],
\end{aligned}
$$

where we note the explicit time dependence of the Liouville operator engendered by the time dependent potential.

Explicitly, the characteristic function has the form

$$
C_{\boldsymbol{\lambda}}\left(x t, x_{0} t_{0}\right)=\left\langle\exp \left(\sum_{n} \lambda_{n} Q_{n}(t)\right) \delta\left(x-f\left(t, x_{0} t_{0}\right)\right)\right\rangle .
$$

Here $f\left(t, x_{0} t_{0}\right)$ is a solution of the Langevin equation (6.27) for a specific noise realisation $\xi(t)$ defining a forward trajectory in configuration space from the initial configuration $x_{0}$ at time $t_{0}$ to the final configuration $x$ at time $t$. Note that a time dependent protocol is acting along the trajectories. We, moreover, introduce the boundary and initial conditions

$$
\begin{aligned}
& C_{\mathbf{0}}\left(x t, x_{0} t_{0}\right)=\left\langle\delta\left(x-f\left(t, x_{0} t_{0}\right)\right)\right\rangle=P\left(x t, x_{0} t_{0}\right), \\
& C_{\boldsymbol{\lambda}}\left(x t, x^{\prime} t\right)=\delta\left(x-x^{\prime}\right) .
\end{aligned}
$$

Since the Liouville operator has an explicit time dependence we solve (6.29) by iteration. Introducing for convenience the operator notation $\hat{C}_{\boldsymbol{\lambda}}\left(t t^{\prime}\right) \equiv C_{\boldsymbol{\lambda}}\left(x t, x^{\prime} t^{\prime}\right), \hat{I}=\delta\left(x-x^{\prime}\right)$, and $\hat{L}_{\boldsymbol{\lambda}}(t) \equiv L_{\boldsymbol{\lambda}}\left(x x^{\prime}, t\right)=L_{\boldsymbol{\lambda}}(x t) \delta\left(x-x^{\prime}\right)$ we obtain

$$
\hat{C}_{\boldsymbol{\lambda}}\left(t t^{\prime}\right)=\hat{I}+\int_{t^{\prime}}^{t} d t^{\prime \prime} \hat{L}_{\boldsymbol{\lambda}}\left(t^{\prime \prime}\right)+\int_{t^{\prime}}^{t} d t^{\prime \prime} \int_{t^{\prime}}^{t^{\prime \prime}} d t^{\prime \prime \prime} \hat{L}_{\boldsymbol{\lambda}}\left(t^{\prime \prime}\right) \hat{L}_{\boldsymbol{\lambda}}\left(t^{\prime \prime \prime}\right)+\cdots .
$$


Next, using time ordering, i.e., $T\left(\hat{L}(t) \hat{L}\left(t^{\prime}\right)\right)=\hat{L}(t) \hat{L}\left(t^{\prime}\right)$ for $t>t^{\prime}$ and $T\left(\hat{L}(t) \hat{L}\left(t^{\prime}\right)\right)=$ $\hat{L}\left(t^{\prime}\right) \hat{L}(t)$ for $t^{\prime}>t$, we have more compactly [38]

$$
\hat{C}_{\boldsymbol{\lambda}}\left(t t^{\prime}\right)=T\left[\exp \left(\int_{t^{\prime}}^{t} d t^{\prime \prime} \hat{L}_{\boldsymbol{\lambda}}\left(t^{\prime \prime}\right)\right)\right]
$$

or expanded in matrix form

$$
C_{\boldsymbol{\lambda}}\left(x t, x^{\prime} t^{\prime}\right)=T\left[\exp \left(\int_{t^{\prime}}^{t} d t^{\prime \prime} \hat{L}_{\boldsymbol{\lambda}}\left(t^{\prime \prime}\right)\right)\right]\left(x x^{\prime}\right) .
$$

The fundamental symmetry of the Liouville operator only refers to the configuration coordinate $x$ and does not involve the time dependence of the potential. Thus, we obtain as in (2.16) the symmetry

$$
L_{\left\{\lambda_{n}\right\}}\left(x x^{\prime}, t\right)=\exp \left(-\beta_{k}\left(U(x t)-U\left(x^{\prime} t\right)\right)\right) L_{\left\{\beta_{n k}-\lambda_{n}\right\}}\left(x^{\prime} x, t\right),
$$

or in operator form, introducing $\exp \left(\beta_{k} \hat{U}(t)\right) \equiv \exp \left(\beta_{k} U(x t)\right) \delta\left(x-x^{\prime}\right)$,

$$
L_{\left\{\lambda_{n}\right\}}(t)=\exp \left(-\beta_{k} \hat{U}(t)\right) \hat{L}_{\left\{\beta_{n k}-\lambda_{n}\right\}}^{*}(t) \exp \left(\beta_{k} \hat{U}(t)\right)
$$

where $*$ indicates the transposed (hermitian conjugate) operator. 


\section{n-th reservoir}

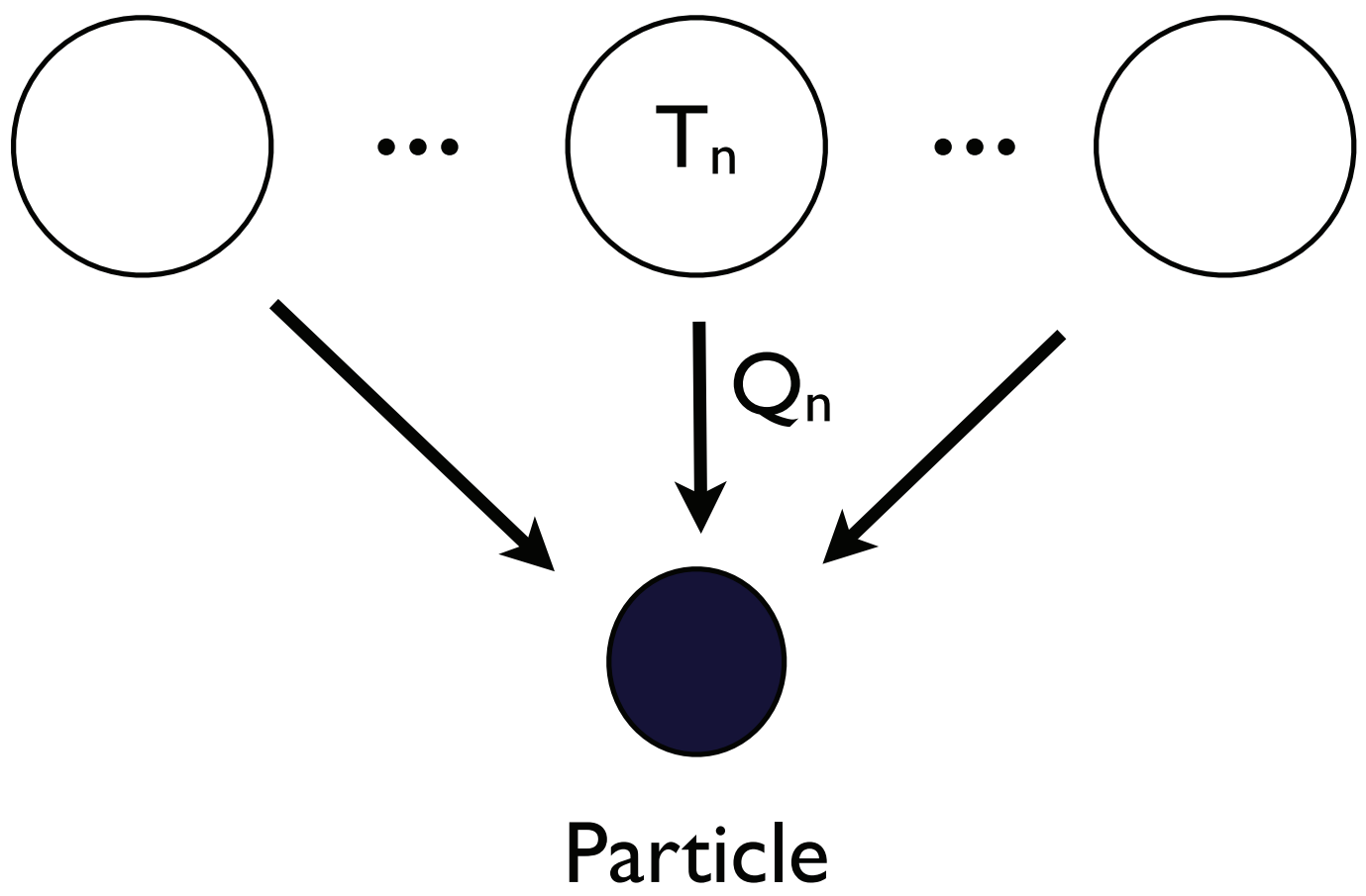

FIG. 1: We depict the configuration corresponding to a single degree of freedom, a particle, coupled to several reservoirs. The temperature of the $n$-th reservoir is maintained at $T_{n}$. The heat transferred to the particle from the n-th reservoir is denoted $Q_{n}$.

[1] E. Trepagnier, C. Jarzynski, F. Ritort, G. Crooks, C. Bustamante, and J. Liphardt, Proc. Natl. Acad. Sci. USA 101, 15038 (2004).

[2] D. Collin, F. Ritort, C. Jarzynski, S. B. Smith, I. T. Jr, and C. Bustamante, Nature 437, 231 (2005).

[3] C. Tietz, S. Schuler, T. Speck, U. Seifert, and J. Wrachtrup, Phys. Rev. Lett. 97, 050602 (2006).

[4] V. Blickle, T. Speck, L. Helden, U.Seifert, and C. Bechinger, Phys. Rev. Lett. 96, 070603 (2006). 
[5] G. Wang, E. Sevick, E. Mittag, D. J. Searles, and D. J. Evans, Phys. Rev. Lett. 89, 050601 (2002).

[6] A. Imparato, L. Peliti, G. Pesce, G. Rusciano, and A. Sasso, Phys. Rev. E 76, 050101R (2007).

[7] A. Imparato, F. Sbrana, and M. Vassalli, Europhys. Lett 82, 58006 (2008).

[8] F. Douarche, S. Joubaud, N. B. Garnier, A. Petrosyan, and S. Ciliberto, Phys. Rev. Lett. 97, 140603 (2006).

[9] N. Garnier and S. Ciliberto, Phys. Rev. E 71, 060101(R) (2007).

[10] A. Imparato, P. Jop, A. Petrosyan, and S. Ciliberto, J. Stat. Mech p. P10017 (2008).

[11] S. Ciliberto, A. Imparato, A. Naert, and M. Tanase, Phys. Rev. Lett. 110, 180601 (2013).

[12] S. Ciliberto, A. Imparato, A. Naert, and M. Tanase, J. Stat. Mech p. P12014 (2014).

[13] J. V. Koski, T. Sagawa, O.-P. Saira, Y. Yoon, A. Kutvonen, P. Solinas, M. Mttnen, T. AlaNissila, and J. P. Pekola, Nat. Phys. 9, 644 (2013).

[14] C. Jarzynski, Phys. Rev. Lett. 78, 2690 (1997).

[15] J. Kurchan, J. Phys. A 31, 3719 (1998).

[16] G. Gallavotti, Phys. Rev. Lett. 77, 4334 (1996).

[17] G. E. Crooks, Phys. Rev. E 60, 2721 (1999).

[18] G. E. Crooks, Phys. Rev. E 61, 2361 (2000).

[19] U. Seifert, Phys. Rev. Lett. 95, 040602 (2005).

[20] U. Seifert, Europhys. Lett 70, 36 (2005).

[21] D. J. Evans, E. G. D. Cohen, and G. P. Morriss, Phys. Rev. Lett. 71, 2401 (1993).

[22] D. J. Evans and D. J. Searles, Phys. Rev. E 50, 1645 (1994).

[23] G. Gallavotti and E. G. D. Cohen, Phys. Rev. Lett. 74, 2694 (1995).

[24] J. L. Lebowitz and H. Spohn, J. Stat. Phys. 95, 333 (1999).

[25] P. Gaspard, J. Stat. Phys. 117, 599 (2004).

[26] A. Imparato and L. Peliti, Phys. Rev. E 74, 026106 (2006).

[27] R. van Zon and E. G. D. Cohen, Phys. Rev. Lett. 91, 110601 (2003).

[28] R. van Zon, S. Ciliberto, and E. G. D. Cohen, Phys. Rev. Lett. 92, 130601 (2004).

[29] R. van Zon and E. G. D. Cohen, Phys. Rev. 67, 046102 (2003).

[30] R. van Zon and E. G. D. Cohen, Phys. Rev. E 69, 056121 (2004).

[31] T. Speck and U. Seifert, Eur. Phys. J. B 43, 521 (2005).

[32] M. Esposito and C. V. den Broeck, Phys. Rev. Lett. 104, 090601 (2010). 
[33] H. C. Fogedby and A. Imparato, J. Stat. Mech. p. P05015 (2011).

[34] H. C. Fogedby and A. Imparato, J. Stat. Mech. p. P04005 (2012).

[35] G. B. Cuetara, M. Esposito, and A. Imparato, Phys. Rev. E 89, 052119 (2014).

[36] H. Risken, The Fokker-Planck Equation (Springer-Verlag, Berlin, 1989).

[37] N. G. van Kampen, Stochastic Processes in Physics and Chemistry (North-Holland, Amsterdam, 1992).

[38] J. Zinn-Justin, Quantum Field Theory and Critical Phenomena (Oxford University Press, Oxford, 1989).

[39] N. N. Lebedev, Special functions and their applications (Dover Publications, New York, 1972).

[40] H. Touchette, Phys. Rep. 478, 1 (2009).

[41] F. den Hollander, Large Deviations, vol. 14 (American Mathematical Society, Providence, R.I., 2000).

[42] P. Visco, J. Stat. Mech. p. P06006 (2006).

[43] J. Farago, J. Stat. Phys. 107, 781 (2002).

[44] B. Derrida and E. Brunet, Einstein aujourd'hui (EDP Sciences, Les Ulis, 2005).

[45] K. Sekimoto, Prog. Theo. Phys. (Suppl.) 130, 17 (1998).

[46] L. Landau and E. Lifshitz, Quantum Mechanics (Pergamon Press, Oxford, 1959). 